\title{
LETTER \\ Regulation of Parallel Converters with Respect to Stored Energy and Passivity Characteristics
}

\author{
Takashi HIKIHARA ${ }^{\dagger a)}$, Member and Yuzo MURAKAMI ${ }^{\dagger}$, Nonmember $^{\circ}$
}

\begin{abstract}
SUMMARY A regulation of converters connected in parallel is discussed considering their stored energy and passivity characteristics. From the viewpoint of stored energy, a new regulation method to conserve and share the stored energy can be found. The energy stored in inductors and capacitors is transferred to loads so that the load keeps the energy dissipation constant. Though numerical simulation, the method is validated for a parallel converter system.

key words: parallel converter, energy, passivity, PWM, transient
\end{abstract}

\section{Introduction}

Recently, power converters have been used as key devices in dispersed power sources. In a dispersed power system, a parallel connection seems an appropriate structure to achieve these goals reliably and to adjust the variations of input voltages for constant output voltage. However, the conventional design method related to converters is inefficient.

In this decade, a control theory based on energy stored in a system was proposed; this scheme targets the passivity characteristics and is applied to various systems [1][4]. The converter models based on this theory are physically rational and their dynamics are easy to depict [2]. On the other hand, in the design of a power converter's control system, the role of power and energy was discussed from the standpoint of power-factor control [5]. It was shown that the energy in the converter circuits is a substantial state variable related to power flow and a strategy was proposed. The general formulation was given by Sira-Ramirez et al. [6] and Escobar et al. [7] on the basis of control and dynamics. The Hamiltonian formulation, in particular, is important for grasping the dynamics of the energy flow in converter circuits. The concept of passivity is applicable to both linear and nonlinear system. Passivity physically corresponds to the energy of the system. The converter is an electric circuit with active and passive elements. Consider a one-port circuit consisting of passive elements with the energy stored in the one-port circuit $E\left(t_{0}\right)$. The total supplied energy in $\left[t_{0}, t\right]$ is $W\left(t_{0}, t\right)$. If $\forall t_{0}$ and $t>t_{0}, E\left(t_{0}\right)+W\left(t_{0}, t\right) \geqslant 0$ is satisfied, then the circuit is passive [8].

In 1980s, Takegaki and Arimoto proposed a control strategy for a finger manipulator based on passivity [9].

\footnotetext{
Manuscript received September 13, 2010.

Manuscript revised November 8, 2010.

${ }^{\dagger}$ The authors are with the Department of Electrical Engineering, Kyoto University, Kyoto-shi, 616-8510 Japan.

a)E-mail: hikihara@kuee.kyoto-u.ac.jp

DOI: 10.1587/transfun.E94.A.1010
}

They introduced a dissipation term into the system in order to preserve the asymptotic feature of energy. It was called "passivity-based control". The researchers are encouraged to control their systems taking into consideration the energy of system [10]. Assuming that each subsystem maintains passivity characteristic, a system with series, parallel, or mixed connections will satisfy the overall passivity characteristics while maintaining impedance of the subsystems.

In this letter, we apply the passivity characteristics [9], [10] to stabilize the converters connected in parallel. If the converters connected in parallel satisfy their passivity characteristics individually, it is expected that each subsystem maintains energy without any mutual interaction [8]. The characteristics seem applicable to the design of the converters coupled in parallel for their safety. In the numerical simulation, a Cuk converter is selected for estimation of the control method because it is well know to have an appropriate characteristic in parallel operation [11].

\section{Regulation of Single Ćuk Converter with Respect to Passivity}

\section{1 Ćuk Converter and Its Dynamics [11]}

Here the operation of a Ćuk converter is explained. The operation is common to other pulse width modulation (PWM) converters. The circuit configuration is shown in Fig. 1. The converter consists of power source $E$, load $R$, inductances $L_{1}$ and $L_{3}$, capacitances $C_{2}$ and $C_{4}$, diode $\mathrm{D}$, and switch $S_{1}$. According to the switching rate of $S_{1}$, the converter can boost or step down the output voltage [12]. The control of the output feedback is usually performed by PWM depending on the difference between the output and reference voltages. D is assumed to be ideal with no conducting resistance. In addition, other passive elements are also ideal. The energy dissipation caused by stray resistances can be separated from the equilibrium energy balance, so that the discussion based on the ideal elements is effective in the control

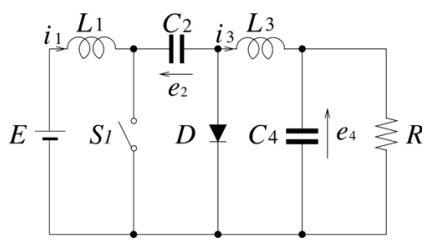

Fig. 1 Configuration of Ćuk converter. 
method's design.

The circuit operation is governed by switching variable $s$ (ON: $s=1$, OFF: $s=0$ ) based on Kirchhoff's law, where $\phi_{1}$ and $\phi_{3}$ denote the magnetic flux stored in inductances $L_{1}$ and $L_{3} . q_{2}$ and $q_{4}$ are the charges stored in $C_{2}$ and $C_{4}$. Here, the state-averaging method can be applied [12]. State variables $\phi_{1}, q_{2}, \phi_{3}$, and $q_{4}$ are averaged in the switching period. Hereafter all the variables are given as their averaged values. When the duty ratio $\mu$ is given by $T_{\text {on }} /\left(T_{\text {on }}+T_{\text {off }}\right)$, where $T_{\text {on }}$ and $T_{\text {off }}$ are the periods of the on and off states, respectively, and $T_{\text {on }}+T_{\text {off }}$ gives the switching period, the continuous circuit relation becomes

$$
\left(\begin{array}{l}
\dot{\phi}_{1} \\
\dot{q}_{2} \\
\dot{\phi}_{3} \\
\dot{q}_{4}
\end{array}\right)=\left(\begin{array}{cccc}
0 & -(1-\mu) & 0 & 0 \\
(1-\mu) & 0 & \mu & 0 \\
0 & -\mu & 0 & -1 \\
0 & 0 & 1 & 0
\end{array}\right)\left(\begin{array}{c}
\frac{\phi_{1}}{L_{1}} \\
\frac{q_{2}}{C_{2}} \\
\frac{\phi_{3}}{L_{3}} \\
\frac{q_{4}}{C_{4}}
\end{array}\right)+\left(\begin{array}{c}
E \\
0 \\
0 \\
-\frac{e_{4}}{R}
\end{array}\right)
$$

\subsection{Hamiltonian Form}

A Hamiltonian form is extended to a practical system with canonical standard equations [10], [13]:

$$
\left\{\begin{array}{l}
\dot{\boldsymbol{q}}=\frac{\partial \mathcal{H}}{\partial \boldsymbol{p}}, \dot{\boldsymbol{p}}=-\frac{\partial \mathcal{H}}{\partial \boldsymbol{q}}+\boldsymbol{u} \\
\boldsymbol{y}=\dot{\boldsymbol{q}}
\end{array}\right.
$$

where $\mathcal{H}$ denotes the Hamiltonian with $\mathbf{q}$ the state variable, $\mathbf{p}$ the momenta, $\mathbf{u}$ a control vector, and $\boldsymbol{y}$ the system output.

The stored energy of Ćuk converter $\mathcal{H}$ depends on the current $\left(i_{1}, i_{3}\right)$ in the inductors $L_{1}$ and $L_{3}$ and the voltage $\left(e_{2}\right.$, $e_{4}$ ) on the capacitors $C_{2}$ and $C_{4}$ as follows:

$$
\mathcal{H}=\frac{1}{2}\left\{L_{1} i_{1}^{2}+C_{2} e_{2}^{2}+L_{3} i_{3}^{2}+C_{4} e_{4}^{2}\right\}
$$

Here $\boldsymbol{q}$ and $\boldsymbol{p}$ have the relationship; $\dot{\boldsymbol{q}}=\left(i_{1}, e_{2}, i_{3}, e_{4}\right)^{T}$ and $\dot{\boldsymbol{p}}=\left(e_{1}, i_{2}, e_{3}, i_{4}\right)^{T}$. Physically they are the variables for electric charge of capacitors and the flux of inductors. However, the physical values are not used in the analysis. The suffix $T$ denotes transpose. According to Eq. (2), $\mathcal{H}$ satisfies the following relationship:

$$
\left\{\begin{array}{l}
\frac{\partial \mathcal{H}}{\partial \boldsymbol{p}}=\dot{\boldsymbol{q}}=\left(i_{1}, e_{2}, i_{3}, e_{4}\right)^{T}, \\
\frac{\partial \mathcal{H}}{\partial \boldsymbol{q}}=-\dot{\boldsymbol{p}}+\boldsymbol{u}=\left(\begin{array}{c}
E-e_{1} \\
-i_{2} \\
-e_{3} \\
-\frac{e_{4}}{R}-i_{4}
\end{array}\right),
\end{array}\right.
$$

The relationships are also the definitions of $e_{i}$ and $i_{i}$ for $i=$ $1, \ldots 4$, where in Eq. (2), the control input vector $\boldsymbol{u}$ is given as $\boldsymbol{u}=\left(E, 0,0,-e_{4} / R\right)^{T}$. Then, the derivative of $\mathcal{H}$ is

$$
\dot{\mathcal{H}}=\frac{\partial \mathcal{H}}{\partial \boldsymbol{q}} \dot{\boldsymbol{q}}+\frac{\partial \mathcal{H}}{\partial \boldsymbol{p}} \dot{\boldsymbol{p}}=\boldsymbol{u}^{T} \boldsymbol{y}=E i_{1}-\frac{e_{4}^{2}}{R} .
$$

The integration of Eq. (5) from $-\infty$ to $t$ gives the following relationship:

$$
\mathcal{H}(t)-\mathcal{H}(-\infty)=\int_{-\infty}^{t} E i_{1} \mathrm{~d} \tau-\int_{-\infty}^{t} \frac{e_{4}{ }^{2}}{R} \mathrm{~d} \tau
$$

This relationship implies that the stored energy is equal to the input energy minus the energy consumed by the resistance. Then, Eq. (6) satisfies the passivity characteristics without depending on the switching ratio $\mu$ [6]. Through this formulation, the idealized discussion of converter circuits suggests that non-idealized circuits, which imply dissipative circuits, will be stable from the standpoint of passivity. Therefore, the discussion based on the passivity can have a role in designing the system regulation method.

The Cuk converter satisfies the passivity characteristics, such that the dynamics under energy dissipation controlled by a scalar input $u$ can be discussed under a constant scalar input $E[3]$.

\section{Derivation of Regulation Method with Respect to Energy}

3.1 Regulation of Converter to Maintain Its Passivity Characteristics

If the Hamiltonian $\mathcal{H}$ has a minimum at $\boldsymbol{q}=\overline{\boldsymbol{q}}$ and an appropriate dissipation control exists, $\overline{\boldsymbol{q}}$ becomes asymptotically stable [13]. Similar to physical systems, the Ćuk converter formalized by a Hamiltonian canonical form is expected to be stable through the regulation of the dissipation under a constant voltage input $E$.

Here, the variation system for the averaged converter model is considered. The equilibria of the coordinate $\boldsymbol{p}$ is set at $\boldsymbol{p}_{0}\left(=\left(\phi_{1 d}, q_{2 d}, \phi_{3 d}, q_{4 d}\right)\right.$ in the system. Then, the Hamiltonian of the variation system is given by the following:

$$
\begin{aligned}
\eta=\frac{1}{2} & \left\{\frac{1}{L_{1}}\left(\phi_{1}-\phi_{1 d}\right)^{2}+\frac{1}{C_{2}}\left(q_{2}-q_{2 d}\right)^{2}\right. \\
& \left.+\frac{1}{L_{3}}\left(\phi_{3}-\phi_{3 d}\right)^{2}+\frac{1}{C_{4}}\left(q_{4}-q_{4 d}\right)^{2}\right\}
\end{aligned}
$$

The derivative of the Hamiltonian with respect to the variable

$$
\boldsymbol{\xi}=\left(\phi_{1}-\phi_{1 d}, q_{2}-q_{2 d}, \phi_{3}-\phi_{3 d}, q_{4}-q_{4 d}\right)^{T}
$$

gives

$$
\frac{\partial \eta}{\partial \boldsymbol{\xi}}=\left(\begin{array}{c}
i_{1}-i_{1 d} \\
e_{2}-e_{2 d} \\
i_{3}-i_{3 d} \\
e_{4}-e_{4 d}
\end{array}\right),
$$

where the state $\left(i_{1 d}, e_{2 d}, i_{3 d}, e_{4 d}\right)^{T}$ is an ideal periodic steady state of $\left(i_{1}, e_{2}, i_{3}, e_{4}\right)^{T}$. When $\mu_{d}$ is set at the target voltage $e_{d}$ by $\mu_{d}=-e_{d} /\left(E-e_{d}\right)$, the proposed regulation method is obtained. The system model is depicted by Eq. (10). 


$$
\left(\begin{array}{l}
e_{1} \\
i_{2} \\
e_{3} \\
i_{4}
\end{array}\right)=\left(\begin{array}{cccc}
0 & -\left(1-\mu_{d}\right) & 0 & 0 \\
\left(1-\mu_{d}\right) & 0 & \mu_{d} & 0 \\
0 & -\mu_{d} & 0 & -1 \\
0 & 0 & 1 & -\frac{1}{R}
\end{array}\right) \frac{\partial \eta}{\partial \xi}-K \frac{\partial \eta}{\partial \xi} .
$$

The matrix $K$ is a $4 \times 4$ gain matrix whose elements are positive, defined by the design of control. $\dot{\eta}$ is obtained from

$$
\dot{\eta}=\frac{\partial \eta^{T}}{\partial \xi} \dot{\xi}=-\frac{1}{R}\left(e_{4}-e_{4 d}\right)^{2}-\frac{\partial \eta^{T}}{\partial \xi} K \frac{\partial \eta}{\partial \xi}<0
$$

Then, this control input vector $\boldsymbol{u}$ can achieve asymptotic stability to $\boldsymbol{\xi}=(0,0,0,0)^{T}$, that is, the state is converged to $\boldsymbol{p}_{0}$.

\subsection{Regulation by Duty Ratio}

The output of Ćuk converter can be regulated by the duty ratio of switching. However, the original converter has already been regulated by PWM; therefore, the duty ratio must be modulated under the control.

The state equation of the Ćuk converter is revised based on Eq. (10).

$$
\begin{aligned}
\left(\begin{array}{l}
e_{1} \\
i_{2} \\
e_{3} \\
i_{4}
\end{array}\right)= & \left(\begin{array}{cccc}
0 & -\left(1-\mu_{d}\right) & 0 & 0 \\
\left(1-\mu_{d}\right) & 0 & \mu_{d} & 0 \\
0 & -\mu_{d} & 0 & -1 \\
0 & 0 & 1 & -\frac{1}{R}
\end{array}\right)\left(\begin{array}{l}
i_{1} \\
e_{2} \\
i_{3} \\
e_{4}
\end{array}\right) \\
& +\left(\begin{array}{l}
1 \\
0 \\
0 \\
0
\end{array}\right) E-K\left(\begin{array}{c}
i_{1}-i_{1 d} \\
e_{2}-e_{2 d} \\
i_{3}-i_{3 d} \\
e_{4}-e_{4 d}
\end{array}\right)
\end{aligned}
$$

Here we assume that the operation of the converter is governed by $\mu^{\prime}$. We set the elements of $K$ as $k_{\mathrm{ij}}(>0)$ in Eq. (12). Then, four types of $\mu^{\prime}$, that is $\mu_{1}^{\prime}, \mu_{2}^{\prime}, \mu_{3}^{\prime}$, and $\mu_{4}^{\prime}$, are obtained by using $\mu_{d}, k_{\mathrm{ij}}(>0)$, and state variables.

$$
\left\{\begin{array}{l}
\mu_{1}^{\prime}=\mu_{d}-k_{12}+k_{12} \frac{e_{2 d}}{e_{2}}, \\
\mu_{2}^{\prime}=\mu_{d}-k_{21}+k_{21} \frac{i_{1 d}}{i_{1}}, \\
\mu_{3}^{\prime}=\mu_{d}-k_{23}+k_{23} \frac{i_{3 d}}{i_{3}}, \\
\mu_{4}^{\prime}=\mu_{d}-k_{32}+k_{32} \frac{e_{2 d}}{e_{2}} .
\end{array}\right.
$$

That is, the solution for $\mu^{\prime}$ is not unique. The arbitrary selection implies that the conditions for maintaining energy are not rigid.

The weighted arithmetic mean of $\mu^{\prime}=\sum_{i}^{4} a_{i} \mu_{i}^{\prime}$ might give an appropriate value of $\mu^{\prime}$ at an operating state. $\mu^{\prime}$ must be optimized for practical applications. Here, we select one of the simple arrithmetic average. The optimization method will be explored in our future design of circuits. We adopt $\mu^{\prime}$ by a simple arithmetic average of $\mu_{2}^{\prime}$ and $\mu_{3}^{\prime}$ as $\mu^{\prime}=\frac{1}{2}\left(\mu_{2}^{\prime}+\right.$ $\left.\mu_{3}^{\prime}\right)$ in the following numerical simulations.

\section{Numerical Simulation of Control on Parallel Con- verters}

In this section, the aforementioned method is applied to a

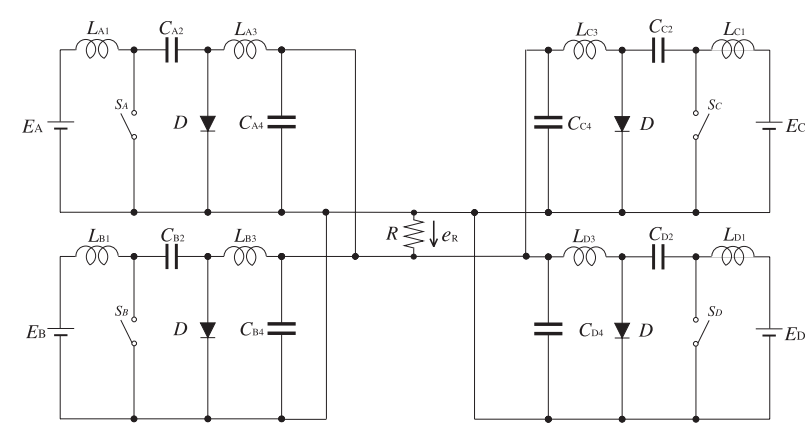

Fig. 2 Ćuk converters connected in parallel to single load.

Table 1 Circuit parameters in the simulation.

\begin{tabular}{|cc||cc|}
\hline $\mathrm{A}$ & & $\mathrm{B}$ & \\
\hline$L_{A 1}$ & $100 \mu \mathrm{H}$ & $L_{B 1}$ & $300 \mu \mathrm{H}$ \\
\hline$C_{A 2}$ & $150 \mu \mathrm{F}$ & $C_{B 2}$ & $200 \mu \mathrm{F}$ \\
\hline$L_{A 3}$ & $150 \mu \mathrm{H}$ & $L_{B 3}$ & $200 \mu \mathrm{H}$ \\
\hline$C_{A 4}$ & $200 \mu \mathrm{F}$ & $C_{B 4}$ & $50 \mu \mathrm{F}$ \\
\hline$E_{A}$ & $12 \mathrm{~V}$ & $E_{B}$ & $8 \mathrm{~V}$ \\
\hline \hline $\mathrm{C}$ & & $\mathrm{D}$ & \\
\hline$L_{C 1}$ & $200 \mu \mathrm{H}$ & $L_{D 1}$ & $100 \mu \mathrm{H}$ \\
\hline$C_{C 2}$ & $450 \mu \mathrm{F}$ & $C_{D 2}$ & $350 \mu \mathrm{F}$ \\
\hline$L_{C 3}$ & $300 \mu \mathrm{H}$ & $L_{D 3}$ & $400 \mu \mathrm{H}$ \\
\hline$C_{C 4}$ & $200 \mu \mathrm{F}$ & $C_{D 4}$ & $150 \mu \mathrm{F}$ \\
\hline$E_{C}$ & $6 \mathrm{~V}$ & $E_{D}$ & $10 \mathrm{~V}$ \\
\hline
\end{tabular}

Ćuk converter based on the rules given by $\mu^{\prime}$. The individually controlled converters are connected in parallel. This configuration is used for the low-voltage-high-current converter characteristics. On the basis of the control rules, the convergence of the output voltage and stored energy is numerically confirmed under external disturbances.

\section{(a) Circuit settings and conditions}

In order to confirm the possibility of regulation by the proposed method in a parallel operation, four Ćuk converters, indexed as A, B, C, and D, are connected in parallel as shown in Fig. 2. The circuit parameters are set as in Table 1.

The power from several power sources is fed to the same load through different converters. Here the load is set at $R=1 \Omega$. When the current fed from the converters maintains a constant ratio, a step voltage disturbance in one of the input power sources and a step change of the target voltage are given to confirm the response of the regulation method, respectively. The simulation is performed by a fourth-order Runge-Kutta algorithm.

\section{(b) Transient response to step change of target voltage}

The transient behavior in the converters approached a steady state by $t=0.1 \mathrm{~s}$. Then the target voltage was shifted from $e_{d}=-5 \mathrm{~V}$ to $e_{d}=-4.5 \mathrm{~V}$. The total Hamiltonian and the output voltage also converge as shown in Figs. 3(a) and 3(b). Figiure 3(b) shows the transient behavior of each Hamiltonian $H_{A}, H_{B}, H_{C}$, and $H_{D}$. We found that each Hamiltonian converges. The change in the duty ratio is shown in Fig. 3(c). The dissipation of the Hamiltonian is regulated by the duty ratio so that the output voltage con- 


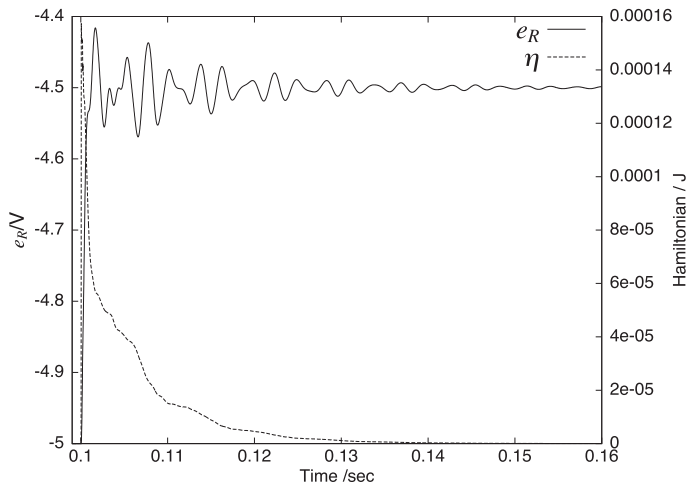

(a) Output voltage and Hamiltonian

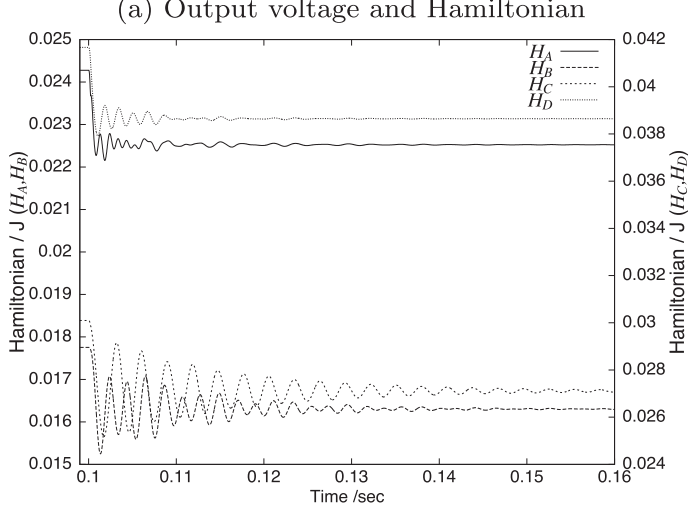

(b) Each regulated Hamiltonian

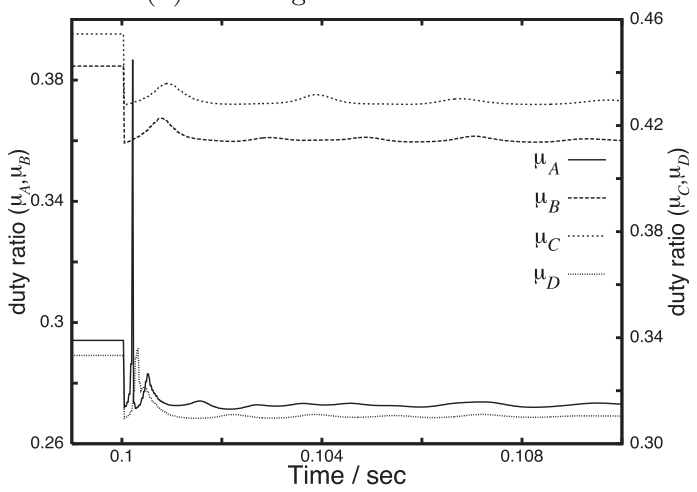

(c) Duty ratio of each converters

Fig. 3 Response to a step change of demand voltage.

verges to the target value.

(c) Transient response to step change of input voltage

The transient response of the same parallel converters is examined for a step change of input voltage. The dispersed power sources consist of different types of sources, including batteries, photo-voltaic systems, and so on. If the converters can regulate themselves without any fluctuation of their output, the method of parallel converters is effective. A simulation is performed to estimate the response characteristics to a step change of input voltage of the converter A. $E_{A}$, changes at $t=0.1 \mathrm{~s}$ from $12 \mathrm{~V}$ to $10.8 \mathrm{~V}$, a $10 \%$ change of the input voltage. The total Hamiltonian and the output voltage still converge to the original target as shown in Figs. 4(a) and 4(b). The change in duty ratio is

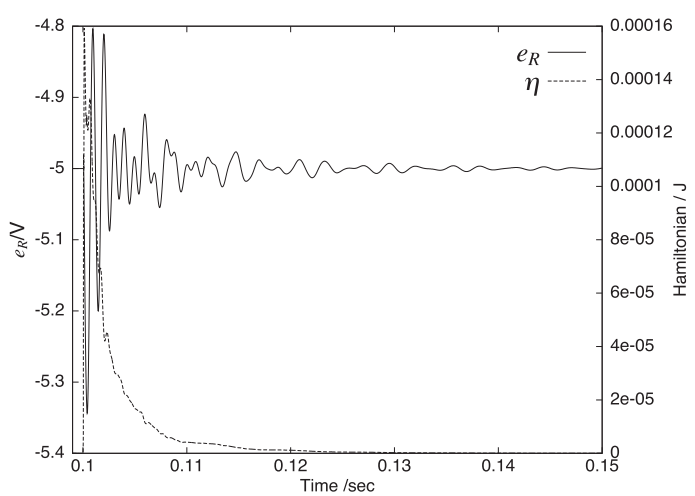

(a) Output voltage and Hamiltonian

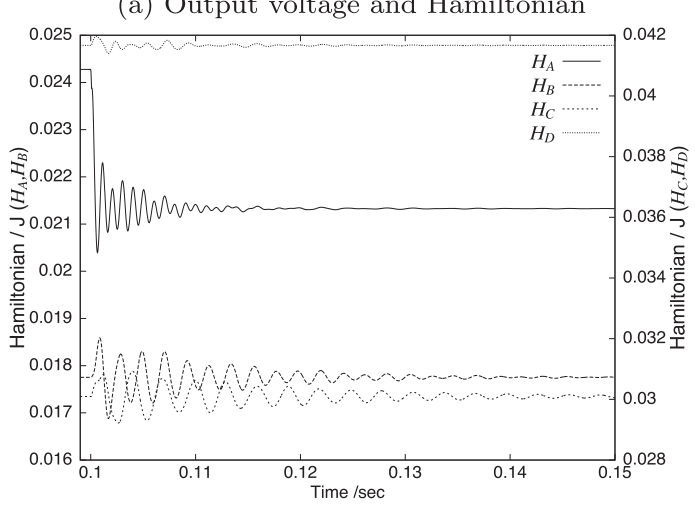

(b) Each regulated Hamiltonian

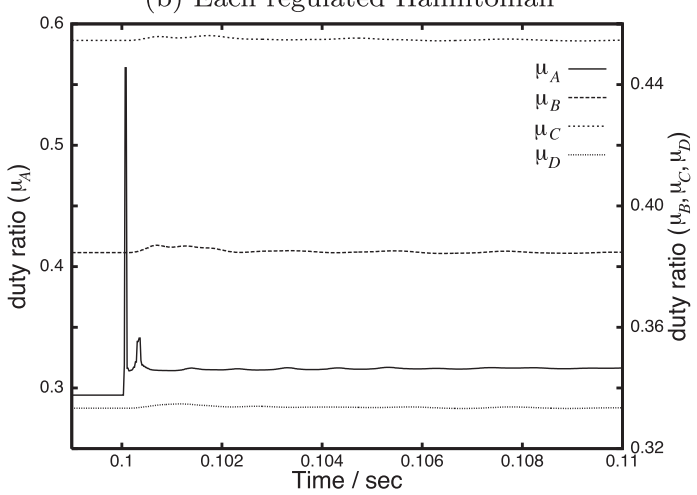

(c) Duty ratio of each converters

Fig. 4 Response to a step change of input voltage.

in Fig. 4(c). Except for converter A, the converged values of the Hamiltonians are the same as before the step change. After the convergence, there are no mutual interactions caused by the change. The regulation is performed by the change in the duty ratio mainly in converter A.

\section{Concluding Remarks}

In this letter, we proposed a method based on passivity for regulation of parallel converters with respect to the stored energy of the circuit. The Hamiltonian of a system deviated around the target state can be stabilized through the regulation of the system dissipation. Numerical simulations show that the proposed method can regulate the output values even if the converters are connected in parallel without any inter- 
action after the convergence.

In parallel converters, the variation of the Hamiltonian describes the variational dynamics around the target system. At the same time, the individual Hamiltonians show independent asymptotic behavior due to the passivity-preserving regulation.

We found that the control method based on passivity could keep the system stable even in the transient operation. The variances of Hamiltonian were confirmed the stability through the proposed regulation method. Practically, the input level of the power converters is inherently limited. In the case, the sudden change of input voltage must be sufficiently covered by other converters as if the converters are cooperating. The redundancy of the parallel connected power sources may help in reducing the number of units.

\section{Acknowledgement}

This research work is partially supported by National Institute of Communications Technology (NICT). We acknowledge for the funding.

\section{References}

[1] K.J. Astrom and K. Furuta, "Swinging up a pendulum by energy control," Automatica, vol.36, pp.287-295, 2000.

[2] R. Ortega, A.J. van der Schaft, I. Mareels, and B. Maschke, "Putting energy back in control," IEEE Control Syst. Mag., vol.21, no.2, pp.18-33, April 2001.
[3] R. Ortega, A.J. van der Schaft, B. Maschke, and G. Escobar, "Interconnection and damping assignment passivity-based control of portcontrolled Hamiltonian systems," Automatica, vol.38, pp.585-596, 2002.

[4] A.J. van der Schaft, "Port-controlled Hamiltonian systems: Towards a theory for control and design of nonlinear physical systems," SICE, vol.39, no.2, pp.91-98, 2000.

[5] M.O. Eissa, S.B. Leeb, G.C. Verghese, and A.M. Stanković, "Fast controller for a unity-power-factor PWM rectifier," IEEE Trans. Power Electron., vol.11, no.1, pp.1-6, 1996.

[6] H. Sira-Ramirez, R.A. Perez-Moreno, R. Ortega, and M. GariciaEsteban, "Passivity-based controllers for the stabilization of DC-toDC power converters," Automatica, vol.33, no.4, pp.499-513, 1997.

[7] G. Escobar, A.J. van der Schaft, and R. Ortega, "A Hamiltonian viewpoint in the modeling of switching power converters," Automatica, vol.35, pp.445-452, 1999.

[8] C.A. Desoer and E.S. Kuh, Basic Circuit Theory, Chap.19, McGrawHill, 1969.

[9] M. Takegaki and S. Arimoto, "A new feedback method for dynamic control of manipulations," Trans. ASME, J. Dynamic Systems, Measurement, and Control, vol.102, pp.119-125, 1981.

[10] K. Fujimoto, K. Sakurama, and T. Sugie, "Trajectory tracking control of port-controlled Hamiltonian systems via generalized canonical transformations," Automatica, vol.39, no.12, pp.2059-2069, 2003.

[11] S. Ćuk and R.D. Middlebrook, A new optimum topology switching DC-to-DC converter, IEEE Power Electronics Specialist Conference Record, pp.160-179, 1977.

[12] L.G. Kassakian, M.F. Schlecht, and G.C. VErghese, Principles of Power Electronics, Addidon-Wesley, 1991

[13] V.I. Arnold, Mathematical methods of classical mechanics, Springer-Verlag, 1989. 